\title{
Utilizing the Personas Concept as a Basis for Modeling Use Cases of a Learning Analytic Application
}

\author{
Margarita Elkina, Andreas Pursian \\ Hochschule für Wirtschaft und Recht Berlin, Germany
}

\begin{abstract}
We describe several user scenarios and usabilityrequirements for the development of the tool, which shall provide detailed information on user behavior inside e-Learning environments. The development takes place within the framework of the Project LeMo (Learning process monitoring on personalizing and non-personalizing e-Learning environments). The tool is based on the analysis, interpretation and categorization of over 80 questions provided by our business partners. Detailed knowledge of our target group and a differentiated view on user requirements are mandatory for the success of the project. Therefore we apply the concept of Persona in order to identify the potential users of the tool and to determine their aims and expectations with regards to the application. Furthermore, a scenario based concept is well suited for providing insights into navigation paths preferred by a specific group of users. In addition we present the first results of the problemcentered interviews which were conducted among our partners to further elaborate on user scenarios and usability-requirements. Finally we summarize our ongoing and upcoming work in the field of information visualization.
\end{abstract}

\section{Introduction}

LeMo is an interdisciplinary research project situated in the field of learning analytics. We utilize data processing technics (data-mining, aggregation, etc.) to extract implicit usage pattern and other relevant information from usage data of personalizing and non-personalizing e-Learning environments.

Nowadays more and more universities and institutions of further education offer blended or distance learning courses to their students in order to meet the needs of increased mobility and lifelong learning concepts. Therefore, a considerable number of elaborated e-Learning platforms like Moodle, Blackboard, Clicks (eCampus), etc. is utilized to provide learning materials, discussion forums and (self-assessment environments anywhere and anytime. While students are using these kinds of eLearning platforms, they leave their digital footsteps in the form of log files. These log files provide the data basis for the LeMo project and will be further analyzed and processed with the goal of creating meaningful visual representations.

Within the LeMo project we focus on two different types of common e-Learning environments: personalizing platforms as well as non-personalizing platforms (user can stay anonymous and choose her/himself the learning sequence).

\section{2. e-Learning spaces}

Many well-known e-Learning environments belong to the category of so-called personalizing learning platforms [1]. An important characteristic of this type of learning platforms is the obligatory presence of a personalized user account. Through this account, the students have access to the learning system and all resources which are enabled for them. Moreover, this is accompanied by the possibility to trace all interactions of the learner with the learning system.

The collected usage data form the basis for the evaluation of learning analytics systems. Educational "tools" such as assignments and tests can therefore be used individually and adaptively to determine the learning progress of a specific learner. This can be done as part of a self-evaluation process of the students themselves as well as through the supervising teacher. Based on the knowledge gained, the teacher can intervene if support seems appropriate.

Personalizing platforms therefore have the advantage that the learning environment can be tailored to the individual needs of the learner. Both the selection of resources relevant to them as well as the provision of communication tools can be realized with ease. On the other hand, the student hereby is often limited to a specific part of the learning resources available at the platform. Personalizing eLearning platforms are used often at universities but spread more and more in the field of higher or elementary schools as well. Well-known examples are Blackboard [2], Moodle [3], Ilias [4] and Clix [5].

On the other hand, the category of nonpersonalizing learning platforms is defined by the absence of an individual user account. Access to these systems is therefore typically anonymous. This 
leads to the implication that nearly all learning resources are fully available to all learners. Unlike personalizing systems and due to the fact, that an individual learner is not easy to identify during an eLearning session, specific learning patterns are difficult to identify from the point of view of the operator.

Non-personalizing e-Learning platforms often implement an alternative way of structuring eLearning contents. Alongside course-based approaches also encyclopedic approaches - similar e.g. to Wikipedia - are used as well. Helpful guidelines for students through the often huge amount of e-Learning material are provided by learning paths [6]. These serve as a guideline for the learner to achieve the aimed learning objectives. Learning paths in non-personalizing e-Learning systems are most often identical for all learners.

A known representative of the latter type of platforms in Germany is the encyclopedic structured learning system Chemgapedia [7].

\section{Project goals}

The main goals for the prototype development are: a data source agnostic backend (therefore, the prototype will support different major e-Learning environments rather than a specific one) which provides the foundation for comparisons between similar courses, as well as a dynamic and adaptive graphical user interface for the visual representation of educational usage data with a strong emphasis on an intuitive and easy usability of the application.

\section{Partners}

LeMo is a collaborative project and is carried out by three German Universities of Applied Sciences: the Berlin School of Economics and Law (HWR), the University of Applied Sciences (HTW) and Beuth University of Applied Sciences (BTH). A strong practical element is ensured by our business partners: I-MC AG (Clix Campus) is a global service and technology provider in the field of e-Learning, FIZ Chemie is an expert in chemical technical information, eLeDia is a specialist in Moodle based e-Learning solutions, Babble is an international provider of online-based language courses, and the BBW College is a private university in Berlin with a strong focus on e-Learning.

\section{Requirement analysis}

The methodological starting point of the project is a list of over 80 questions provided by our business partners, for example:
- How long, how often, in which order and within which time period was a specific learning resource used by a scholar?

- How long does a user session take?

- How many scholars used a given resource at a given time within the time interval $x$ ?

- What kind of relationship exists between the usage of the resources and the results of the tests?

- Is it possible (perhaps via the background semantic analysis) to recover the degree of the student satisfaction from his/her behavior?

- Which of the courses are characterized by the high breakup rate, in proportion to the calls of the learning content?

- Is it possible to draw from comparison and analysis of the user behavior the conclusions on his/her educational level?

Since these questions come from the field of both personalizing and non-personalizing platforms, they turn for us into a number of different research problems: Who are the main stakeholders of such an application and which individual requirements do they have? How should a graphical user interface be designed, in order to best suit the needs and requirements of our heterogeneous target group? What kind of graphical representation is the most appropriate for a specific monitoring requirement (e.g. path analysis, natural language processing, etc.)?

\section{Target group analysis}

While the LeMo project puts strong emphasis on the creation of an easy to understand and easy to use user interface (see 9. Design Examples), detailed knowledge of our target group and a differentiated view on user requirements are mandatory for the success of the project.

"If not all contributors of a system [are] determined in time, this can increase the likelihood that a number of requirements could not be recognized." [8, p. 94].

The project focuses on a wide and heterogeneous target group which includes not only online tutors and professors at universities, but also marketing and product development staff of organizations providing personalized as well as non-personalized e-Learning offerings. Researchers interested in further investigating of user behavior within both types of eLearning platforms, belong to our target group as well. To get a more precise representation of this heterogeneity, we took the decision to utilize the persona concept.

The persona (lat., mask) concept was initially proposed by Alan Cooper [9][14]. This concept provides a methodological toolbox for the precise 
and differentiated definition of target groups and their individual requirements during the process of application development. It was widely discussed by usability engineers and adopted by bigger companies [10] to implement user centered design (UCD) concepts in the field of software development.

\section{Personas}

A persona is a fictional character who has a number of concrete attributes like:

- Personal information (e.g. name, age, profession, etc.)

- Personal goals (in general)

- Personal objectives in relation to the application

- Hobbies and personal characteristics

Each persona is a representative of a specific part of the target group and is therefore summarizing a common set of personal attributes and requirements of this group. A persona gains a lot of its value through its detailed description, for example in comparison to archetype user representation methods [11].

Utilizing personas can help the developer to better understand a certain requirement because he/she can put him/herself in the position of a specific persona.

Hence, a scenario based approach is hopefully well suited to provide insights into navigation paths preferred by a specific user group.

\subsection{Persona creation}

In the first step, assumption personas [12, p.188] were created based on our first ideas about the target group. In order to verify and adjust these personas, we conducted partly standardized interviews in combination with an identical online survey with relevant employees of all partner organizations. In this context, usability-aspects as well as individual preferences in relation to the design of the application and user interface, acquire especial significance.

For this small, non-representative survey, five key persons with equally strong but different eLearning background were interviewed. Additionally - to the same extent - results could be collected through an online questionnaire.

Below we present a selection of our questions:

- Which of the following internet (WWW) related terms are familiar to you? (Multiple answers possible)

$\begin{array}{ll}\circ & \text { Widget } \\ \circ & \text { Dashboard } \\ \circ & \text { Drop-Down } \\ \circ & \text { Plugin } \\ \circ & \text { Browser }\end{array}$

\section{- Drag\&Drop \\ ○ None}

- Which of the following statistical terms are familiar to you? (Multiple answers)

$\begin{array}{ll}\circ & \text { Variance } \\ \circ & \text { Median } \\ \circ & \text { Significance } \\ \circ & \text { Average } \\ \circ & \text { Universe } \\ \circ & \text { Expression } \\ \circ & \text { None }\end{array}$

- Will the application probably stay in your personal usage or will it be used by the other team workers/colleagues/partners as well?

- How many people will probably use the application in your organization?

- How would you estimate the potential merits of the application in relation to your personal working context?

- How often will you probably use the application and can you already estimate how long will be every single session?

- The answers to which three most important questions do you expect to obtain with the help of the application? For example:

○ Question 1: Have all exercises been solved correctly?

- Question 2: Which exercises have been most often solved wrong?

- Question 3: Are there anomalies among the assessments of exercises?

- From your point of view, how important is the presence of a graphical representation of statistical estimates by the application?

- From your point of view, how important is the tabularization of statistical estimates by the application?

- What are the absolutely necessary functions you would demand from the application, e.g. Reporting, printing, generation of PDF, etc.?

- Which tools of analysis and/or monitoring tools are you currently using to track user activities in your e-Learning environments?

- When you open your personal start page, what kind of information is the most important / valuable for you? (Only one answer)

a. Overview of all courses / learning paths learning units with a few key figures

b. A few courses / learning paths / learning units with detailed information

c. Information on potentially problematic developments

d. Other (please enter a total of 1 max.)

- Which three of the following application features are the most important for you? 

a. Ease of use
b. Individual customizability of the graphical interface
c. Prompt answers
d. Permanently updated database
e. Easy learnability
f. Broad range of functioning
g. Comparability of results
h. Miscellaneous (please insert up to 3 items )

- Is there an administrator, who would take care of the configuration and functioning of the application?

Some results: $87.5 \%$ of our participants plan to use the application in person. $75 \%$ of the respondents described the potential benefits of the application for their personal work environment as useful or very useful (with one abstention).

Concerning the contents, over $62.5 \%$ of our respondents are interested in the areas of user navigation and learning path analysis. For $62.5 \%$ of the respondents it was also important or very important, that the analysis results can be displayed graphically.

In regard to the desired functionalities, in particular an export function $(67.5 \%)$ for further processing of the analysis data as well as a print functionality (50\%, multiple answers possible) was highly demanded by our respondents.

Based on the results of this survey, we were able to verify and further specify three of our assumption personas and added a fourth one. We did this by classifying similar requirements and grouping our respondents by personal characteristics like professional background and work experience.

While each persona has their individual set of personal characteristics and requirements, we tried to identify potential similarities between them in order to make additional classifications possible. As a result of this effort, we were able to categorize our personas in three user categories: lecturer, domain expert and decision maker.

Thereby one persona is associated with the category Domain expert and Decision maker respectively, while the Lecturer category is represented by two personas. The following descriptions of user categories widely correspond to the description of the associated personas.

\subsubsection{Domain expert}

To the Domain expert belong:

- Users in manager positions with professional competence in the field of elearning

- Users with professional interest in improvement of the e-learning offers

- Users with long experience in configuration and organization of e-learning offers
- Users with irregular practice of the application and interest for detailed information

The purposes of the Domain Expert in relation to the application are:

- He/she expects a clear and informative preparation of employed data;

- He/she would like to be able to analyze graphically the most frequent patterns of movement of the e-Learning courses participants;

- $\mathrm{He} / \mathrm{she}$ would like to be able to compare the data stemming from various periods of time in order to conclude on the efficiency of changes in the e-learning platform;

- $\mathrm{He} / \mathrm{she}$ would like to use graphic overview as well as detailed information in form of tables;

- He/she would like to obtain exact information about users of e-learning platform and their behavior.

\subsubsection{Decision maker}

The Decision maker characterizes:

- Users with a high innovational capacity

- Users without technical background but with far-reaching experience in e-learning as well as in general online services

- Users with regular practice of the application who require fast informative overview about various courses

- Users with high interest in improvement of didactic offers in educational processes

Purposes:

- Users with managerial responsibility

- He/she would like to obtain tips how to improve his/her work in e-learning environment;

- $\mathrm{He} / \mathrm{she}$ would like to obtain tips to how to integrate the passive students into collaborative activities.

\subsubsection{Lecturer 1}

This type of persona characterizes:

- $\quad$ Elder users of age over 50

- Non-technical users with little knowledge in handling of online platforms

- Users from the research and education field who intend to make e-learning useful for their work

- Users with interest for rapid informative overview (without details)

Of course, the "Lecturer" expectations in relation to application are different from the purposes of Domain expert. It is important for this group of users: 
- to obtain feedback from students and statistical data about the usage of e-learning offers;

- to obtain recommendations for improvement of the e-learning offers;

- $\quad$ to analyze the customized statistical data.

\subsubsection{Lecturer 2}

This type of persona characterizes:

- Young users under age of 30 from the periphery of e-learning providers

- Well-educated users with internet skills and broad experience in social networks

- Users with interest for information on usage of existing e-learning offers with the aim of their improvement

- Users with frequent usage of application and interest to detailed information

- Users open to new methods and Purposes: effort in e-learning field

- He/she would like to work with easily usable adaptive application window;

- $\mathrm{He} / \mathrm{she}$ would like to be able to infer the relations between effect of networking and learn success.

Throughout the development process, personas serve as a semi-formal reminder on existing requirements with regard to the user-centered decision-making. Therefore it is essential that the identified personas are well understood by the whole development team, in order to achieve that all development decisions are made with regard to a concrete persona instead of an often diffuse target group or the personal opinion of a single programmer.

\section{Use Cases}

Based on the results of our requirement and target group analysis, we have proposed three main user categories: lecturer, domain expert and decision maker. These user categories have been transformed as actors within a UML - Use Case representation, to further elaborate on the identified requirements. This step builds the bridge to a more formalized view on the requirements and helps to eliminate misinterpretations.

Therefore Use Cases describe the interaction of various external actors with the system [13], they also provide implementation hints for example inheritance information and communication flows.
To separate use cases of different actors, a role model will be implemented in the LeMo application. The goal of this effort is to deliver to every category of user the use cases (functionalities) they need and, at the same time, to build a bridge between persona based usage scenarios and the necessary implementation details [12, p.541][16]. These efforts will help to reduce the complexity of the system - in general - and the user interface - in particular - to the necessary minimum.

To achieve this goal, short usage scenarios for all essential Use Cases from the viewpoint of each particular persona have been designed. They determine how the interaction with the system is supposed to look from the perspective of a persona. In a second step, the persona scenarios were used to to extract relevant use cases, which then were visualized with the help of UML Use Case diagrams. Through this approach, the connection between a formal request and a specific sequence of user actions is established [12, p32] .

Within this paper we can only present a small selection of the identified Use Case scenarios. For this reason, with the following two examples we focus on some basic features of the application.

The first example provides an overview of the common Use Case Customize analysis (Fig. 1). Herewith, we show how we differentiate use cases between the three actor types. Based on the results of our requirement analysis some use cases are only important for a specific actor(s), while they would unnecessarily clutter the user experience for other actors.

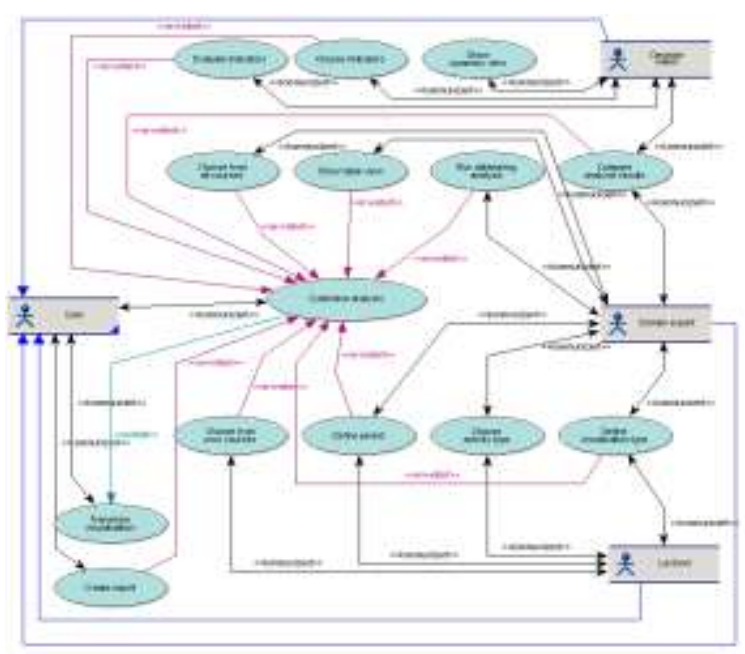

Figure 1. Use Case Customize analysis

Figure 1 gives an overview of the Use Case Customize analysis. Figures $2-4$ provides the relevant magnified cut-outs for each user category 


\subsection{Customize Analysis - Decision Maker}

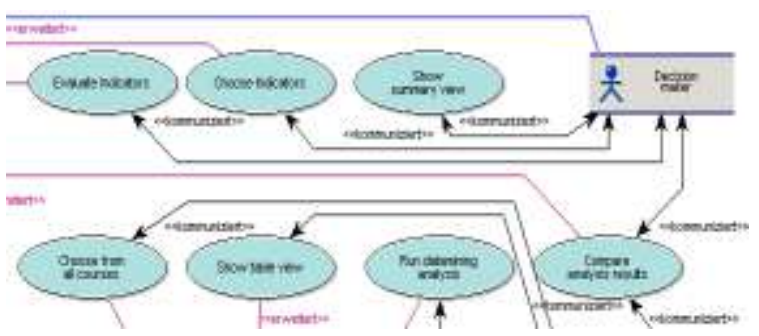

Figure 2. Use Cases Decision Maker

The Decision Maker (see Figure 2) places high emphasis on a quick and effective overview of several key metrics of the e-learning offers and the eLearning system as a whole. In turn, the analysis of individual e-learning resources is of minor importance for him/her.

For this purpose he/she chooses relevant indicators (Choose Indicators) and evaluates them (Evaluate Indicators) based on the analysis results the system provides.

Like the Domain expert, it is equally important for the Decision maker, to identify changes and trends of key figures (indicators) by comparing results of analysis for different time periods (Compare analysis results).

\subsection{Customize Analysis - Domain Expert}

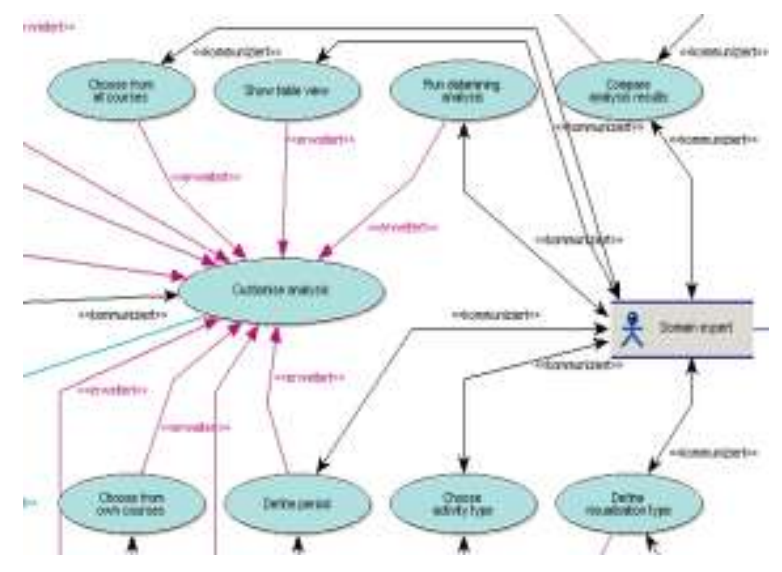

Figure 3. Use Cases Domain Expert

The Domain Expert (Fig. 3) has a strong analytical focus and works regularly with the system. Hence, a comparatively wide range of relevant use case exists for him/her.

Unlike the Decision maker, the Domain Expert usually operates at the level of concrete courses.

In opposite to the Lecturer, he/she has the possibility to access all courses within the system.

Due to his/her stronger technical background he/she is also able to use more advanced analysis functionalities such as data mining analysis (Run data mining analysis).

For the purpose of better evaluation and processing of analytical data in external applications, the Domain expert can use a tabular analysis beside the graphical representation (Show table view) and is able to export this data as well.

\subsection{Customize Analysis - Lecturer}

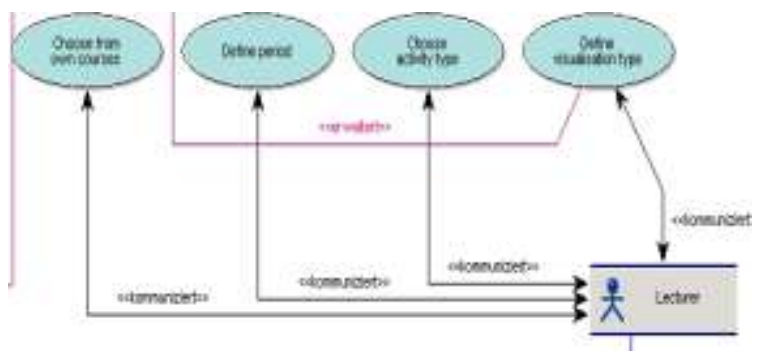

Figure 4. Use Cases Lecturer

The classification Lecturer (Fig. 4) primarily describes lecturers who want to analyze their own courses. For this purpose, he/she shares much of the functionality with the Domain expert.

This concerns basic functionalities like the setup of the analysis which includes the considered time interval (Define period), the selection of the types of activities (eg. activities in the forum, blog, assignments and tests, media, etc.) to be included in the analysis (Choose activity type), as well as the selection of the specific course and the type of the desired visualization (Choose own courses and Choose visualization type).

\subsection{Dashboard}

The second Use Case scenario (Dashboard) exemplifies general purpose use cases, which a user will be faced with while interacting with his/her personal start page.

\section{User Interface}

Man-machine systems (MMS) - like the LeMo application - need to integrate an interface for interacting with the user, in order to present the results produced by the application logic. 


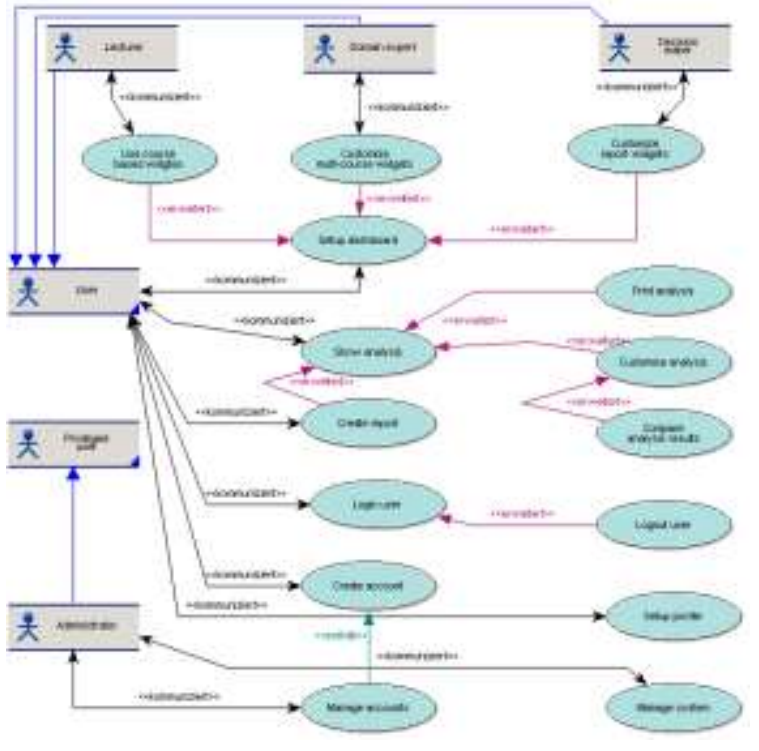

Figure 5. Use Case Dashboard

This interface is used to control the application logic by a defined set of controls that can be used for selective manipulation of the behavior of an application.

On this basis, a layout has been developed, which is characterized by the following assumptions:

- "Content matters", Therefore, getting rid of unnecessary layout elements (banners, etc.)

- Important information must be located within the user visible area of the browser window

- Display only the most necessary controls (context-sensitive)

- Compliance with usability principles in the selection of color and contrast pairs

In addition, in order to achieve good legibility, the user interface is designed to work with different client resolutions (Responsive Design) [14].

We have included an early design Mock-up of our upcoming prototype application. The mock-up (Fig. 8) gives some insights on the design concept of the personal start page (Dashboard).

It shows the basic structure of a user's personal dashboard. The header area at the top of the page contains all context-sensitive control and navigation elements and provides status information about the currently used users account. The space below is completely intended to display the content.

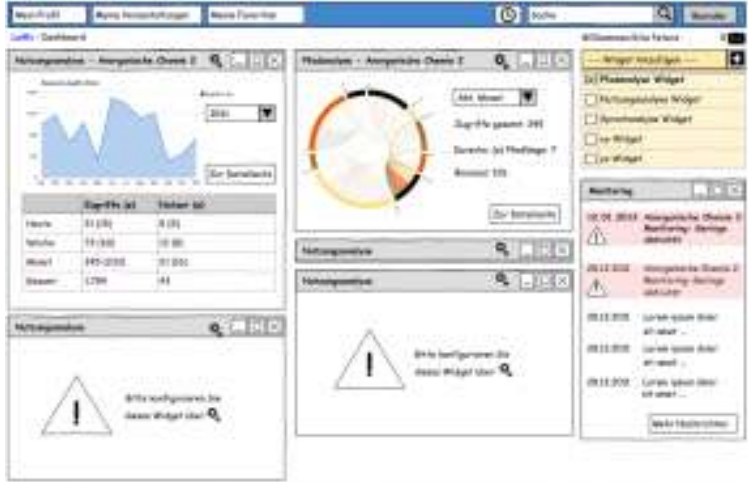

Figure 6. Mock-up Dashboard

\section{Conclusion}

In this paper we have described our approach to the analysis and description of Use Cases based on personas. Our goal is the development of the learning analytic tool called LeMo, which should be applicable for a broad audience in the field of eLearning.

To which extent the demonstrated approach can support a user-centered application development process must be verified by further studies.

\section{References}

[1] Beuster, Liane , Elkina, Margarita , Fortenbacher, Albrecht, Kappe, Leonard, Merceron, Agathe , Pursian, Andreas, Schwarzrock, Sebastian, Wenzlaff, Boris: LeMo - Lernprozessmonitoring auf personalisierenden und nicht personalisierenden Lernplattformen. in Grundfragen Multimedialen Lehrens und Lernens, Von der Innovation zur Nachhaltigkeit, Waxmann, Münster, 2012, p. 425.

[2] Blackboard International, About Blackboard http://www.blackboard.com/About-Bb/Who-We-Are.aspx (20 August 2012).

[3] Moodle, http://de.wikipedia.org/wiki/Moodle, August 2012).

[4] Ilias - Open Source e-Learning, About Ilias, http://www.ilias.de/docu/goto_docu_cat_580.html, August 2012).

[5] IMC Clicks, About IMC http://www.imc.de/germany/en/company/about-us/, (20 August 2012).

[6] Brusilovsky, P., A Framework for Intelligent Knowledge Sequencing and Task Sequencing, pp. 499-506, Proc. Intelligent Tutoring Systems, 1992.

[7] Encyclopedia Chemgapedia, About Chemgaroo, http://www.fiz-chemie.de/en/home/productsservices/education-training.html/ (20 August 2012). 
[8] Oestereich, Bernd et al., OEP-oose Engeneering Process - Vorgehensleitfaden für agile Softwareprojekte, Heidelberg, 2007.

[9] Cooper A., The Inmates are Running the Asylum: Why High-tech products drive us crazy an how to restore the sanity, SAMS, Indianapolis, 1999.

[10] Grudin J. and J. Pruitt, Personas, Participatory Design and Product Development: An Infrastructure for Engagement, Proceedings of Participation and Design Conference, Sweden, 2002, pp. 144-161.

[11] Richter M., "100 Seiten Spezifikation - und was ist die Konsequenz für uns?", OBJEKTspektrum, Requirements Engineering - online edition, June 2008, pp. $1-4$.

[12] Pruitt, J. S. and T. Adlin, The Persona Lifecycle Keeping People in Mind Throughout Product Design, Morgan Kaufmann Publishers - Elsevier, San Francisco, 2006.

[13] Sommerville Ian, Software Engineering, Pearson, Munich, 2012.

[14] Marcotte, Ethan (May 25, 2010). "Responsive Web Design", http://www.alistapart.com/articles/responsiveweb-design (20 August 2012).

[15] Holtzblatt, K., J. Burns Wenell, and S. Wood. Rapid Contextual Design: A How-To Guide to Key Techniques for User-Centered Design. San Francisco: Morgan Kauffman, 2004.

[16] Oestereich, Bernd, Analyse und Design mit UML 2.1 - Objektorientierte Softwareentwicklung. 8. Edtion, Munich/Vienna, 2006. 